\title{
Near Critical States of Random Dirac Fermions
}

\author{
Y. Morita and Y. Hatsugai \\ Department of Applied Physics, University of Tokyo, 7-3-1 Hongo Bunkyo-ku, Tokyo 113, Japan
}

(Received 2 May 1997)

\begin{abstract}
Two-dimensional random Dirac fermions are studied numerically. They are realized on a square lattice by the $\pi$-flux model with random hopping. It preserves a symmetry denoted by $\{\mathcal{H}, \gamma\}=0$ in an effective field theory. Although it belongs to the orthogonal ensemble, the zero-energy states do not localize but become critical. The density of states vanishes as $\sim E^{\alpha}$ and the exponent $\alpha$ changes with strength of the randomness (the critical line). Rapid enhancement of the Thouless number is observed near the zero energy. The level-spacing distribution is also investigated, which is consistent with the existence of the critical states at $E=0 . \quad$ [S0031-9007(97)04538-9]
\end{abstract}

PACS numbers: 72.15.Rn, 05.30.Fk, 71.10.Fd

Dirac fermions often appear in condensed matter physics, for example, a transition between different quantum Hall states [1-3], two-dimensional graphite sheets [4], a mean-field theory of the $t-J$ model (" $\pi$-flux state") [5] and $d$-wave superconductors [6]. It is then natural to investigate what happens when disorder is included. Random Dirac fermions in a two-dimensional space were investigated by several groups [6-12]. Possible appearance of nonlocalized states, critical states, in random Dirac fermions was pointed out in [7]. Recently, this disordered critical state was realized in a lattice model, where it was crucial to preserve a symmetry denoted by $\{H, \gamma\}=0$ with a $4 \times 4$ matrix $\gamma$ in an effective field theory [8] (see below).

In this paper, we study the random Dirac fermions numerically beyond the zero-energy states. In order to realize the massless Dirac fermions on a two-dimensional lattice, we use a tight-binding model on a square lattice with half a flux quantum (" $\pi$ flux") per plaquette, which is described by the Hamiltonian

$$
H_{\text {pure }}=\sum_{\langle i, j\rangle} c_{i}^{\dagger} t_{i j} c_{j}+\text { H.c. },
$$

where the summation is over the nearest-neighbor bonds. The hopping matrix elements are given by $t_{j+\hat{x}, j}=(-1)^{j_{y}}$ and $t_{j+\hat{y}, j}=1$, where $j=\left(j_{x}, j_{y}\right), \hat{x}=(1,0)$, and $\hat{y}=$ $(0,1)$. In the momentum space, the Hamiltonian is rewritten as

$$
H_{\text {pure }}=2 \sum_{\mathbf{k}} \psi_{\mathbf{k}}^{\dagger}\left(\begin{array}{cc}
\cos k_{y} & \cos k_{x} \\
\cos k_{x} & -\cos k_{y}
\end{array}\right) \psi_{\mathbf{k}},
$$

where the summation is over the magnetic Brillouin zone $[-\pi, \pi) \times[0, \pi)$ and $\psi_{\mathbf{k}}^{\dagger}=\left(c_{\left(k_{x}, k_{y}\right)}^{\dagger}, c_{\left(k_{x}, k_{y}+\pi\right)}^{\dagger}\right)$. There are two energy bands $E(\mathbf{k})= \pm 2 \sqrt{\cos ^{2} k_{x}+\cos ^{2} k_{y}}$ on the magnetic Brillouin zone. They touch at two momenta, $\mathbf{k}^{1}=\left(k_{x}^{1}, k_{y}^{1}\right)=(\pi / 2, \pi / 2)$ and $\mathbf{k}^{2}=\left(k_{x}^{2}, k_{y}^{2}\right)=$ $(-\pi / 2, \pi / 2)$. Near the degeneracies $\mathbf{k}^{i} \quad(i=1,2)$, they behave as $E(\mathbf{k}) \approx \pm 2 \sqrt{\left(k_{x}-k_{x}^{i}\right)^{2}+\left(k_{y}-k_{y}^{i}\right)^{2}}$ $(i=1,2)$. Define continuum variables $\Psi^{\dagger}(x, y)=$ $\left(\psi_{1}^{\dagger}(x, y), \psi_{2}^{\dagger}(x, y), \psi_{3}^{\dagger}(x, y), \psi_{4}^{\dagger}(x, y)\right) \quad$ by $\quad c_{j} \sim a \times$ $\left[i^{j_{x}+j_{y}} \psi_{1}(x, y)+i^{j_{x}-j_{y}} \psi_{2}(x, y)+i^{-j_{x}+j_{y}} \psi_{3}(x, y)+\right.$ $\left.i^{-j_{x}-j_{y}} \psi_{4}(x, y)\right]$, where $a$ is the lattice spacing and $x=a j_{x}, y=a j_{y}$. Then the Hamiltonian becomes in the continuum limit $(a \rightarrow 0) \quad \mathcal{H}_{\text {pure }}=2 i \times$ $\int d \mathbf{x} \Psi^{\dagger}(\mathbf{x})\left[\left(\sigma_{3} \otimes \sigma_{1}\right) \partial_{x}+\left(I \otimes \sigma_{3}\right) \partial_{y}\right] \Psi(\mathbf{x})$. Therefore our lattice model includes doubled massless Dirac fermions.

There are several subtleties for the massless Dirac fermions. When the Fermi energy lies at zero energy, that is, all the negative energy eigenstates are filled, the Hall conductivity $\sigma_{x y}$ is ill defined. An infinitesimal mass determines the $\sigma_{x y}$ in the continuum theory [13]. A similar phenomenon also occurs in a lattice model where an infinitesimal next-nearest-neighbor hopping $t^{\prime}$ opens a gap and the $\sigma_{x y}$ is given by $t^{\prime} /\left|t^{\prime}\right|$ [3]. Therefore the massless Dirac fermions are at a quantum phase-transition point between different quantum Hall states.

Let us consider the effect of randomness in the hopping matrix elements. We set $t_{j+\hat{x}, j}=(-)^{j_{y}}+\delta t_{j+\hat{x}, j}$ and $t_{j+\hat{y}, j}=1+\delta t_{j+\hat{y}, j}$, where $\delta t_{j+\hat{x}, j}$ and $\delta t_{j+\hat{y}, j}$ are random variables and taken at random with constant probability from $[-W, W]$. As discussed below, we focus on the role of the symmetry denoted by $\{\mathcal{H}, \gamma\}=0$. Therefore we do not consider diagonal disorder, which breaks the symmetry. It should be noted that this model preserves the time-reversal symmetry and belongs to the orthogonal ensemble. Another example of the orthogonal ensemble, Dirac fermions with diagonal disorder, was studied in $[9,10]$ and it was suggested that all the eigenstates localize, which is consistent with the scaling theory of the Anderson localization [14]. In the case of the random-hopping model, however, it was found that the zero-energy states do not localize but become critical [8]. A similar phenomenon was found at the band center of the quantum Hall states [15]. In the quantum Hall states, however, the time-reversal symmetry is broken and the system belongs to a different universality class, the unitary ensemble. In [8], it was confirmed that parameters for the critical states form a critical line in the parameter space of the Hamiltonian, which is connected to pure 
massless Dirac fermions at zero energy. We consider that the stability of the zero-energy states against random hopping matrix elements is due to a symmetry of our Hamiltonian. The random hopping matrix elements preserve the symmetry in contrast to the diagonal disorder. In the language of the lattice Hamiltonian (1), the symmetry means that the transformation $c_{j} \rightarrow(-1)^{j_{x}+j_{y}} c_{j}$ induces a sign change of the Hamiltonian. Thus the eigenstates always appear in pairs with energies $E$ and $-E$. The corresponding transformation in the continuum Hamiltonian is given by $\mathcal{H}_{\text {pure }} \rightarrow \gamma^{\dagger} \mathcal{H}_{\text {pure }} \gamma=-\mathcal{H}_{\text {pure }}$, where $\gamma=\sigma_{1} \otimes \sigma_{1}$. Since the random hopping matrix elements preserve the symmetry, the continuum Hamiltonian $\mathcal{H}$ for the random-hopping model also satisfies $\{\mathcal{H}, \gamma\}=\mathcal{H} \gamma+\gamma \mathcal{H}=0$. Thus, taking the lowest order in derivatives, we obtain the following form as a possible Hamiltonian for the effective field theory

$$
\mathcal{H}=\mathcal{H}_{\text {pure }}+\sum_{i=1}^{4} \int d \mathbf{x} a_{i}(\mathbf{x}) \Psi^{\dagger}(\mathbf{x}) \gamma^{i} \Psi(\mathbf{x}),
$$

where $\gamma^{1}=\sigma_{2} \otimes I, \quad \gamma^{2}=\sigma_{1} \otimes \sigma_{2}, \quad \gamma^{3}=-\sigma_{2} \otimes \sigma_{1}$, $\gamma^{4}=I \otimes \sigma_{2}$, and $a_{i}(x, y)(i=1, \ldots, 4)$ are random variables.

In this paper, we study random Dirac fermions numerically beyond the zero-energy states. We diagonalize the Hamiltonian for finite squares of size $L^{2}=20^{2}, 30^{2}, 40^{2}$, and $50^{2}$. Here we note that the symmetry discussed above reduces the dimension of the Hilbert space for the numerical diagonalization, which is half of the system size. To obtain reliable statistics, an ensemble average over $16000,16000,8000$, and 3360 realizations is performed, respectively. The observables are density of states $\rho(E)$, the Thouless number $g(E)$, and the level-spacing distribution $P(s)$.

Let us first discuss the density of states $\rho(E)=$ $\sum_{i} \delta\left(E-E_{i}\right) / L^{2}$. When there is no randomness, the $\rho(E)$ vanishes linearly at zero energy. Recently, whether the density of states is finite or not at zero energy for random Dirac fermions is controversial [11,12]. We note that, although random Dirac fermions are also studied in [11,12], the symmetry of disorder is different from ours. Since the symmetry is crucial in the longdistance behavior of wave functions, it is possible that our system belongs to a different universality class from random Dirac fermions studied in $[11,12]$. The $\rho(E)$ 's for different strengths of randomness are shown in Fig. 1. We obtained the $\rho(E)$ 's for $L=30,40$, and 50. In Fig. 1, however, we show only results for $L=50$, since the finite-size effect in $\rho(E)$ is small. We have fitted the data by the power-law form $\rho(E)=C E^{\alpha(W)}$. Our results support, within the limitations imposed by finitesize effects near $E=0$, the vanishing density of states at zero energy with an anomalous exponent $\alpha(W)$, which depends on strength of the randomness.

Next, in order to reveal the nature of the eigenstates, let us consider the Thouless number $g(E)$. The $g(E)$ is

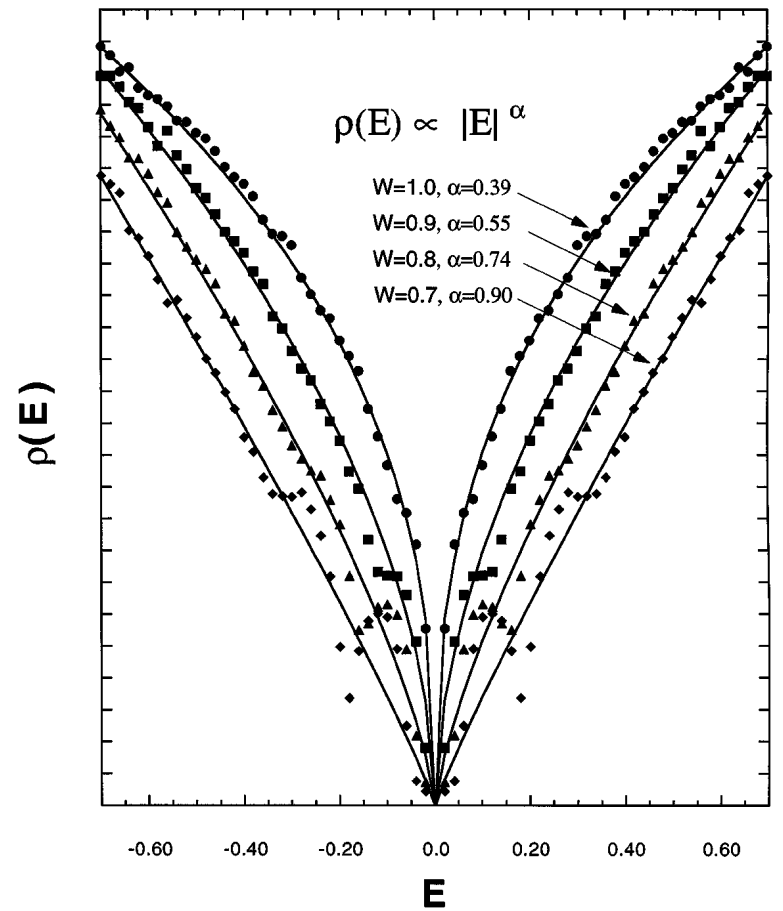

FIG. 1. The density of states $\rho(E)$ (with small imaginary part $\delta=0.02$ ), where $L=50$ and $W=0.7,0.8,0.9$, and 1.0. We have fitted the data by the power-law form $\rho(E)=C E^{\alpha(W)}$, where $\alpha(W)=0.90,0.74,0.55$, and 0.39 for $W=0.7,0.8$, 0.9 , and 1.0 , respectively.

defined by $g(E)=V(E) / \Delta(E)$, where $V(E)$ is an energy shift obtained by replacing the periodic boundary condition by an antiperiodic boundary condition and $\Delta(E)$ is a local mean level spacing near the energy $E$. Numerical results for the $g(E)$ are shown in Fig. 2 with $L=30,40$, and 50, where an ensemble average is performed within an energy window whose center is located at each data point. Rapid enhancement of the $g(E)$ near zero energy is observed in Fig. 2. It suggests that the localization length grows rapidly near zero energy. This is consistent with the existence of critical states at zero energy. One may consider that the zero-energy states are just on the critical point. Then one of the possible scenarios is that the non-zero-energy eigenstates are all off critical and therefore localized. It suggests an exponential dependence of the Thouless number $g(E, L)$. The $g(E, L)$ obtained numerically decreases when the system size increases. It is, however, far from the exponential dependence. In Fig. 2, we have plotted the $g(E, L)$ as a function of $1 / L$. It suggests a power-law form $g(E, L) \propto 1 / L^{\gamma}$ rather than an exponential form $g(E, L) \propto \exp (-L / \xi)$. Although we cannot exclude the possible existence of critical states in a finite energy region, we consider that the non-zero-energy states may be localized in an infinite-size system and a crossover from the power-law form to an exponential form occurs when the system size increases beyond the localization length. The localization length of the eigenstates near zero energy may be large compared to the available 
(a)

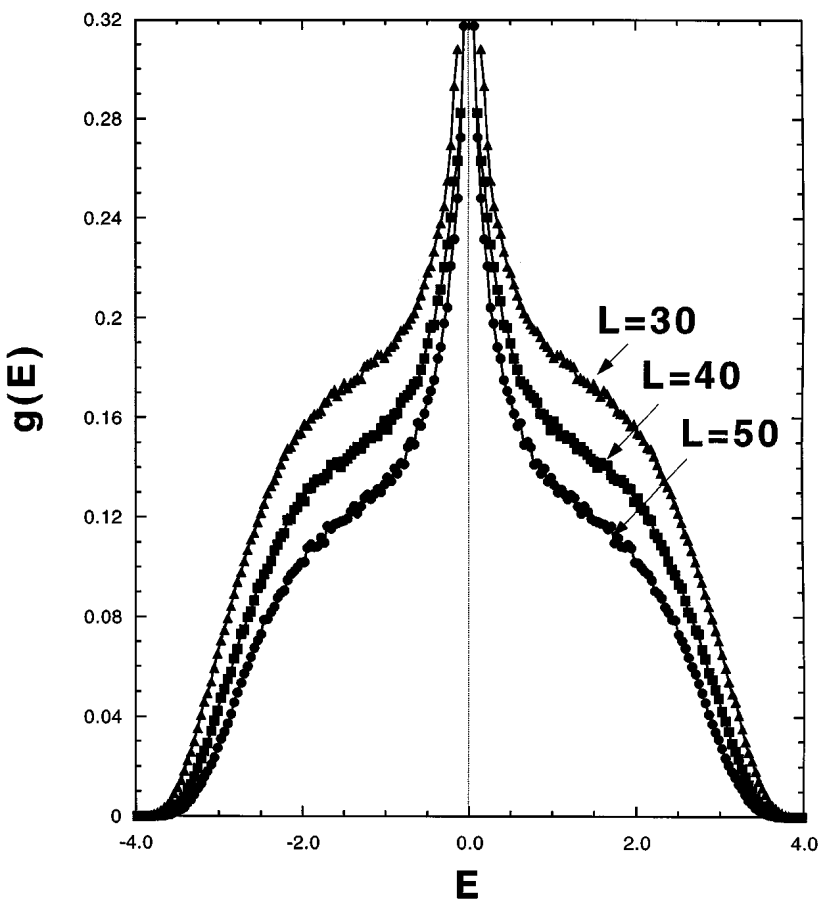

(b)

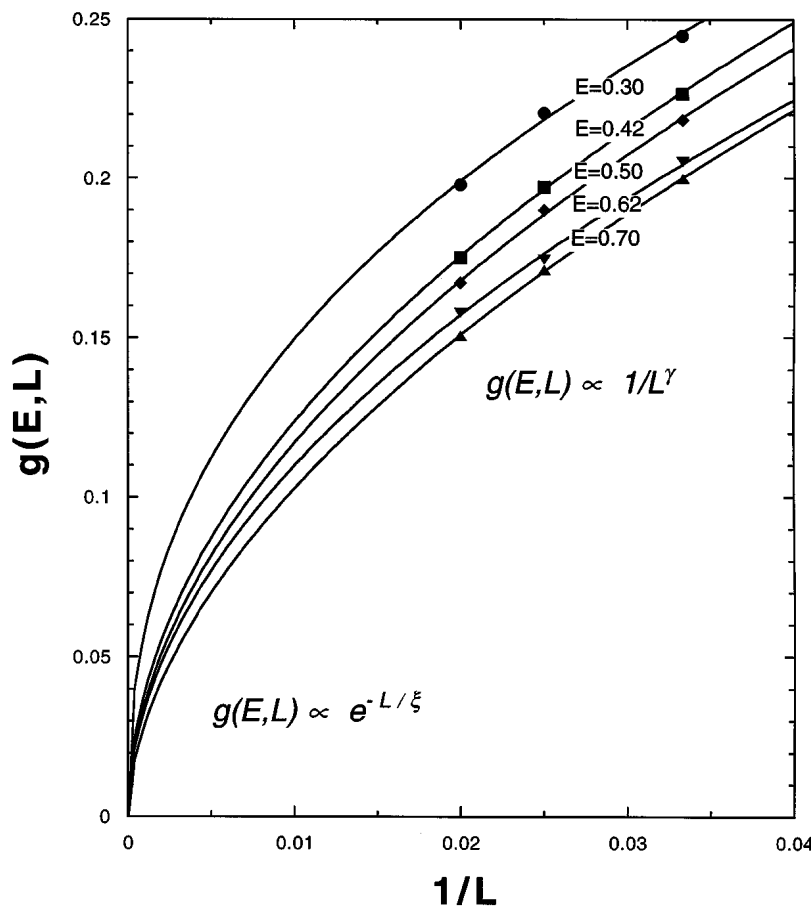

FIG. 2. (a) The Thouless number $g(E)$, where $W=1.0$ and $L=30,40$, and 50 . (b) $1 / L-g(E, L)$ plot for $W=1.0$, $E=0.30,0.42,0.50,0.62$, and 0.70 .

system sizes and we may say that the power-law dependence of the $g(E, L)$ is a critical slowing down in the available finite-size system. This also suggests the existence of the critical state at zero energy.
We have also obtained the level-spacing distribution $P(s)$. The $P(s)$ 's of the normalized energy separation $s=\left|E_{n}-E_{n+1}\right| / \Delta\left(E_{n}\right)$ are shown in Fig. 3, where $E_{n}$ and $E_{n+1}$ are two successive eigenenergies. In general, the $P(s)$ is well described by the Wigner surmise $P(s)=$ $A s^{\beta} \exp \left(-B s^{2}\right)$ in the metallic regime and becomes the Poissonian $P(s)=\exp (-s)$ in the insulating regime. The parameter $\beta$ in the Wigner surmise reflects the symmetry of the Hamiltonian and $\beta=1,2$, and 4 for the orthogonal ensemble, the unitary ensemble, and the symplectic ensemble, respectively. The parameters $A$ and $B$ are determined by $\int_{0}^{\infty} d s P(s)=1$ and $\int_{0}^{\infty} d s s P(s)=$ 1 and, in particular, $A=\pi / 2$ and $B=\pi / 4$ for the orthogonal ensemble. The $P(s)$ characterizes the nature of the eigenstates. States localized in different spatial regions are allowed to lie at the same energy. It means that the energy levels of the localized states distribute independently, which is described by the Poissonian. On the other hand, in metals where the eigenstates are extended, two adjacent energy levels interact strongly, which brings strong energy repulsion and $P(s) \sim s^{\beta}$ near $s=0$, where $\beta$ is determined by the symmetry of the Hamiltonian. The $P(s)$ is well explained by a $2 \times 2$ random matrix model, which is the Wigner surmise. Level statistics near the mobility edge has been studied recently and the appearance of critical level statistics is

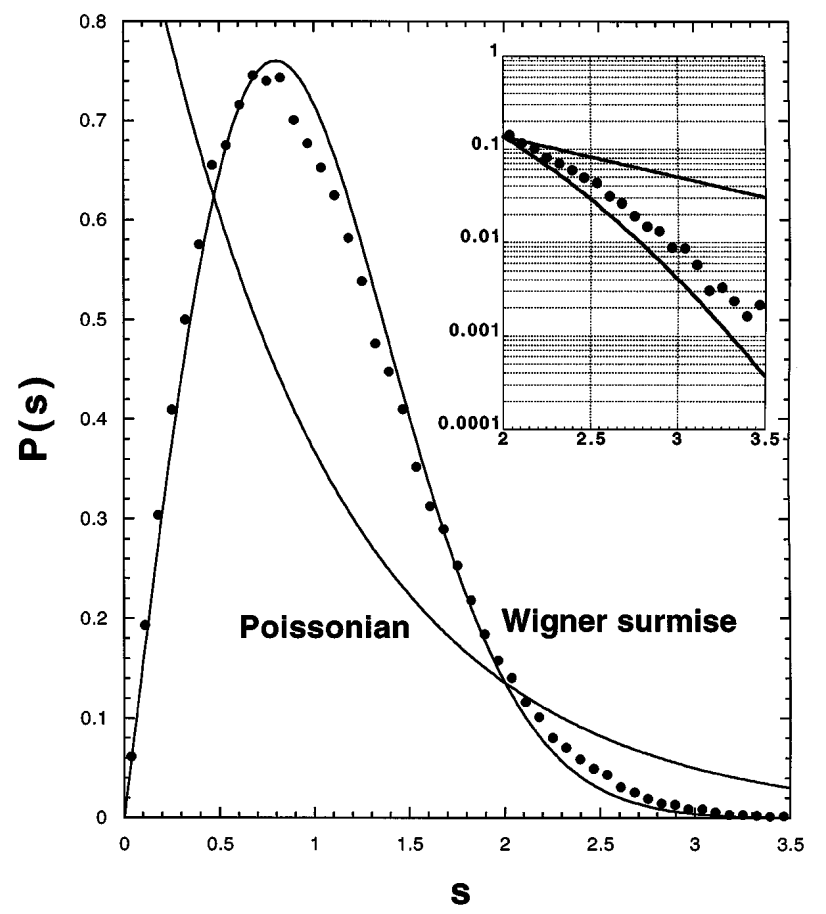

FIG. 3. The level spacing distribution $P(s)$ near zero energy, where $L=50, W=1.0$ and ensemble average is performed within an energy window $[0.1,0.5]$. We confirmed that the finite-size effect is small, comparing the results for $L=50$ with those for $L=20,30$, and 40 . There is no substantial difference between results with energy windows $[0.1,0.3],[0.2,0.4]$, and $[0.3,0.5]$. 
discussed [16]. Several numerical studies on level statistics near the mobility edge were performed for, e.g., the three-dimensional Anderson model [17] and the band center of the quantum Hall states [18], which belong to the orthogonal ensemble and the unitary ensemble, respectively, and it was found that the $P(s)$ 's deviate from both the Wigner surmise and the Poissonian. They exhibit critical behavior, i.e., the $P(s)$ 's do follow the Wigner surmise for small $s$ and they then deviate from it at higher values of $s$ and show stretched exponential decay. The $P(s)$ 's near zero energy for our model are shown in Fig. 3, where the energy window is set $[0.1,0.5][19]$. The location of the energy window is set sufficiently close to zero energy compared to the bandwidth, which corresponds to the energy cut off in the continuum theory. Thus we consider that the system is described by random Dirac fermions. We confirmed that the $P(s)$ 's near zero energy do not seriously depend on the system size and the location of the energy window. Since the location of the energy window is set near zero energy compared to strength of the randomness, the localization lengths are so long that it exceeds the system size. Thus, although the eigenstates may be localized in an infinite-size system, they behave as critical wave functions in a finite-size system. In fact, the $P(s)$ in Fig. 3 deviate from both the Wigner surmise and the Poissonian, and exhibit critical behavior, i.e., the $P(s)$ 's do follow the Wigner surmise for small $s$ and they then deviate from it at higher values of $s$ and show stretched exponential decay. On the other hand, when the location of the energy window is set in other regions, noncritical behavior is found.

In summary, we have studied random Dirac fermions numerically beyond the zero-energy states. Although it belongs to the orthogonal ensemble, the zero-energy states do not localize but become critical. The density of states $\rho(E)$ vanishes as $\sim E^{\alpha}$ near zero energy and the exponent $\alpha$ depends on strength of the randomness $W$. It implies that scaling dimensions of the operators change with strength of the randomness. It is similar to the case of the random gauge-field critical line found in [7]. The existence of the symmetry $\{\mathcal{H}, \gamma\}=0$ is crucial to have the criticality of the zero-energy states. We have studied the nature of the eigenstates using the Thouless number $g(E)$ and the level spacing distribution $P(s)$. As is suggested by the numerical results for the $g(E)$, the localization length grows near zero energy so rapidly that it exceeds the available system size and the observables at nonzero energies exhibits anomalous behavior, that is, a critical slowing down. The $P(s)$ 's near zero energy deviate from both the Wigner surmise and the Poissonian and exhibit critical behavior, as in the case of the quantum Hall states $[18,20]$. It reflects the existence of critical states at zero energy in our model. The random critical line of the two-dimensional Dirac fermions might be unstable in contrast to the critical line of $(1+1)$ dimensional free bosons, Tomonaga-Luttinger liquid, and a global renormalization-group flow for random Dirac fermions is an interesting problem in relation to the quantum Hall effect.

We are grateful to X.G. Wen, M.H. Hettler, K. Ziegler, and P. J. Hirschfeld for valuable communications. Y.H. was supported in part by Grant-in-Aid from the Ministry of Education, Science and Culture of Japan. The computation in this work was done using the facilities of the Supercomputer Center, ISSP, University of Tokyo.

[1] X. G. Wen and Y. S. Wu, Phys. Rev. Lett. 70, 1501 (1993).

[2] W. Chen, M. P. A. Fisher, and Y.S. Wu, Phys. Rev. B 48, 13749 (1993).

[3] Y. Hatsugai and M. Kohmoto, Phys. Rev. B 42, 8282 (1990).

[4] G. W. Semenoff, Phys. Rev. Lett. 53, 2449 (1984).

[5] I. Affleck and J.B. Marston, Phys. Rev. B 37, 3774 (1988).

[6] P. A. Lee, Phys. Rev. Lett. 71, 1887 (1993).

[7] A. Ludwig, M. Fisher, R. Shankar, and G. Grinstein, Phys. Rev. B 50, 7526 (1994); C. Chamon, C. Mudry, and X. G. Wen, Phys. Rev. B 53, 7638 (1996).

[8] Y. Hatsugai, X. G. Wen, and M. Kohmoto, Phys. Rev. B 56, 1061 (1997).

[9] M. P. A. Fisher and E. Fradkin, Nucl. Phys. B251 [FS13], 457 (1985).

[10] Y. Hatsugai and P. A. Lee, Phys. Rev. B 48, 4204 (1993).

[11] A. A. Nersesyan, A. M. Tsvelik, and F. Wegner, Phys. Rev. Lett. 72, 2628 (1994); A. A. Nersesyan and A. M. Tsvelik, Phys. Rev. Lett. 78, 3981 (1997).

[12] K. Ziegler, M. H. Hettler, and P. J. Hirschfeld, Phys. Rev. Lett. 77, 3013 (1996); 78, 3982 (1997).

[13] S. Deser, R. Jackiw, and S. Templeton, Ann. Phys. (N.Y.) 140, 372 (1982); A. Niemi and G. W. Semenoff, Phys. Rev. Lett. 51, 2077 (1983); N. Redlich, Phys. Rev. D 29, 2366 (1984).

[14] E. Abrahams, P.W. Anderson, D. C. Licciardello, and T. V. Ramakrishnan, Phys. Rev. Lett. 42, 673 (1979).

[15] See, for a review, The Quantum Hall Effect, edited by R.E. Prange and S.M. Girvin (Springer, New York, 1990).

[16] V.E. Kratsov, I. V. Lerner, B. L. Altshuler, and A. G. Aronov, Phys. Rev. Lett. 72, 888 (1994).

[17] B. I. Shklovskii, B. Shapiro, B. R. Sears, P. Labrianides, and H. B. Shore, Phys. Rev. B 47, 11487 (1993); S. N. Evangelou, Phys. Rev. B 49, 16805 (1994).

[18] B. Huckstein and L. Schweitzer, Phys. Rev. Lett. 72, 713 (1994); Y. Ono and T. Ohtsuki, J. Phys. Soc. Jpn. 62, 3813 (1993); Y. Avishai, Y. Hatsugai, and M. Kohmoto, Phys. Rev. B 51, 13419 (1995).

[19] We have also calculated $P(s)$ 's for small energy windows near zero energy. We could not obtain systematic differences among them. Therefore we chose a rather wide energy window to obtain reliable statistics.

[20] T. Ando, J. Phys. Soc. Jpn. 52, 1740 (1983); 53, 3101 (1984); 53, 3126 (1984). 